\title{
The Variability of QSOs in the Optical Band
}

\author{
D. Trèvese and A. Bunone
}

Istituto Astronomico Università di Roma "La Sapienza", via G.M. Lancisi 29, I-00161, Roma, Italy

R.G. Kron

Fermi National Accelerator Laboratory, MS 127, Box 500, Batavia, IL 60510

Constraints on the emission mechanism of AGNs can be provided by the variability of their spectral energy distribution (SED). Recently Di Clemente et al. (1996) have shown that the positive correlation of QSO variability with redshift can be due to a hardening of the spectrum in bright phases, coupled with the increase of the rest-frame frequency of the (fixed) observing band, for increasing redshift. Direct evidences of slope changes in the SEDs of a limited number of individual AGNs have been provided by Cutri et al. (1985), Edelson et al. (1990), and Kinney et al. (1991). In the following we present some preliminary results of a direct measure of variations of the SED slope in the complete, magnitude limited, sample of QSOs of the Selected Area 57 (Koo, Kron \& Cudworth 1986; Trèvese et al. 1989 (T89)). The data are derived from two sets of prime focus plates of the SA 57, in the $U, B_{J}, F$ and $N$ bands, obtained with the 4-m telescope at the Kitt Peak National Observatory at two epochs separated by one year. Photometric methods and signal-to-noise optimization are described in T89 and Trèvese et al. (1994). The quasar sample, of $\langle z\rangle \simeq 1.4$, consists of 33 objects from T89, plus the brightest 3 members of the the Bershady, Trèvese and Kron (1998) sample of extended objects selected on the basis of variability. In Figure 1a $\Delta \alpha$ is reported versus the changes $\Delta \log f_{v}$ in the $B_{J}$ band and shows positive correlation, indicating a hardening of the spectrum in the bright phase. A special care is needed to avoid spurious $\Delta \alpha-$ $\Delta \log f_{v}$ correlations (see Massaro \& Trèvese 1996). The correlation coefficient is $\mathrm{r}=0.46$ with a probability $\mathrm{P}(>\mathrm{r})=0.995$, after the exclusion of one deviant point, whose inclusion would produce a higher correlation. The slope of the linear regression of $\Delta \alpha \Delta \log v$ is $b=1.9$. Assuming that the spectra are, dominated by the big blue bump (BBB) in the sampled spectral region, around $\lambda \approx 2000$ $\AA$, we can use the simple approximation of a single black-body spectrum. To check the hypothesis that both the slope and brightness changes are caused by a temperature variations only, we derive the black-body temperatures from the SED slope as deduced from a linear fit of the $\log f_{v}$ v.s. $\log v$ relation, based on the $U, B_{J}$ and $F$ data. The average slope (excluding the two highest and lowest $\mathrm{z}$ objects) is $\langle\alpha\rangle=-0.4 \pm 0.6$ and the average temperature is $T \approx 25000$ K. For a black-body of fixed emitting surface and varying temperature $T$, the changes of the local SED slope and the relevant luminosity variations are related by $(d \alpha / d T) /\left(d \log B_{v} / d T\right) \equiv f(x)$, where $x \equiv h v / k T$. The slope b of the linear regression of $\Delta \alpha$ v.s. $\log f_{v}$ of Figure 1a is compared with the function $f(x)$ 
in Figure 1b, where $\alpha(x)$ for a black-body is also plotted with the range of $\mathrm{x}$
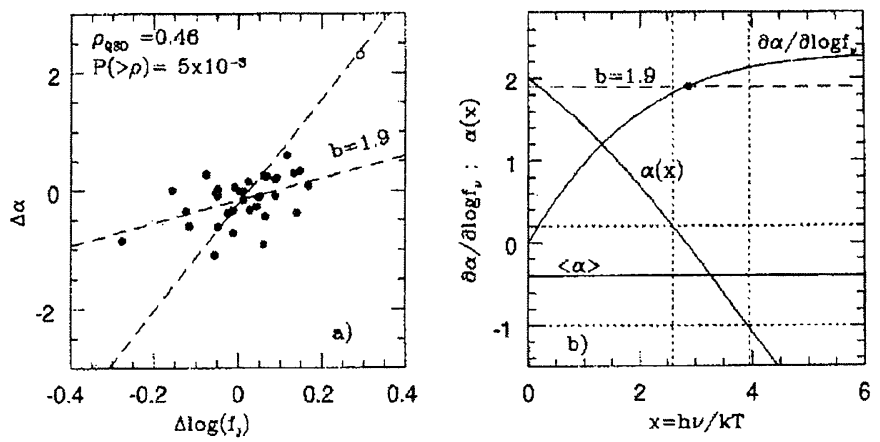

Figure 1. a) Slope changes $\Delta \alpha$ versus $\Delta \log f_{v}$ in the $B_{J}$ band. The straight lines represent the linear regressions, after the exclusion of one deviant point (open circle). b) The functions $f(x) \equiv d \alpha / d \log B_{v}$, and $\alpha(x)$ for a black-body, versus $x \equiv h v / k T$. The values of the slope $\mathrm{b}$ (see Figure 1a), the average $\langle\alpha\rangle$ and the dispersion of the slopes are represented by the horizontal lines. The relevant range of $x$ values is indicated by the vertical dashed lines.

values of the sample determined by the dispersion of $\alpha$. We can conclude that $d \alpha / d \log B_{v}$ and $\alpha$ are consistent for $x \approx 3$. For an average sampling wavelength $\langle\lambda\rangle \approx 2000 \AA$ of the sample, this corresponds to an average temperature $T \approx 2.5 \times 10^{4}$ of a black-body of fluctuating temperature.

The above results show that: i) slope changes of the SED and intensity variation in the optical-UV are at least not inconsistent with temperature variations of an emitting black-body; ii) aside from any specific interpretation, the relation between the variability of slope and intensity found in our complete QSO sample, provides a new constraint on the physical mechanisms responsible of variability.

\section{References}

Bershady, M.A., Trèvese, D., \& Kron, R.C.: 1998, ApJ, 496, 103.

Cutri, R.M., Wisniewski, W.Z., Rieke, G.H., \& Lebofski, H.J.: 1985, ApJ, 296, 423.

Di Clemente, A., Natali, G., Giallongo, B., Trèvese, D., Vagnetti, F.: 1996, ApJ, $463,466$.

Edelson, R.A., Krolik, J.H., Pike, G.F.: 1990, ApJ, 359, 86.

Giallongo, B., Trèvese, D., Vagnetti, F.: 1991, ApJ, 377, 345.

Kinney, A.L., Bohlin, R.C., Blades, J.C., \& York D.G.: 1991, ApJS, 75, 645.

Koo, D.C., Kron, R.G., Cudworth, K.M.: 1986, PASP, 98, 285.

Massaro, E., \& Trèvese, D.: 1996, A\&A, 312, 810. 
Trèvese, D., Pittella, G., Kron;R.G., Koo, D.C., Bershady, M.A.: 1989, AJ, 98, 108. (T89)

Trèvese, D., Kron, R.G., Majewski, S.R., Bershady, M.A., Koo, D.C.: 1994, ApJ, 433, 494. 